\title{
A Comparative Analysis of Performance Gain of 7- nm FinFET over Planer CMOS
}

\author{
Jamal Uddin Ahmed, Sarah Nahar Chowdhury, Mehnaz Haq, Asif Khan, Sadman Shoumik Khan
}

\begin{abstract}
FinFET has been contemplated as a seemly substitute for the conventional CMOS at the nano-scale regime owing to the projection for application in the integrated circuits fabrication due to its extraordinary properties like improved channel controllability, high $\mathrm{ON} / \mathrm{OFF}$ current ratio and reduced short-channel effect. In this paper, circuit simulations of 7-nm FinFET and planer CMOS are comprehensively investigated showing a $34.92 \%$ and $28.79 \%$ increased drain current in $7-\mathrm{nm}$ Fin-FET compared to the existing 22-nm and equivalent $7-\mathrm{nm}$ planer CMOS respectively. A detailed simulation study evaluating the performance of the proposed design is presented exhibiting a $2 X$ increase in drive strength with the increment of fins. Fin thickness of $2.725 \mathrm{~nm}$ along with a height of $10.9 \mathrm{~nm}$ has been used resulting in an $8 \mathrm{X}$ reduction in gate area which is the smallest 7-nm Fin-FET structure yet developed. The indiscriminate variations of the characteristics obtained in various simulations lead to a culmination of shifting to Fin-FET from planer CMOS which is imperative from the prospect of design and manufacture.
\end{abstract} HSpice

Keywords-FinFET, multi-gate FETs, 7-nm, nanoscale,

\section{Introduction}

The steady down-scaling of feature sizes resulting in exponential miniaturization of the CMOS technology in digital circuits has been the driving force for the continual improvement in circuit speed and cost per functionality over the past several decades. Moreover, energy consumption has always been a crucial performance metric for integratedcircuits (ICs) and the voltage down-scaling is quite effectual in reducing the power consumption of ICs which is desirable for some relaxed-performance applications, such as portable wireless devices, medical devices and sensor network nodes [1]. But, with advanced geometry planar FET technologies in the nanoscale regime, such as $20 \mathrm{~nm}$, the source and the drain encroach into the channel, making the leakage current to flow easily which eventually makes it very difficult to turn the transistor off completely resulting in short channel effects, sub-threshold leakage, gate-dielectric leakage, off-state leakage current, delays, power dissipation and device to device variations [2]. Thus, the downscaling of bulk material faces significant challenges below the sub $20-\mathrm{nm}$ that are inhibiting the overall device performance. But, currently, these concerns

Jamal Uddin Ahmed, Sarah Nahar Chowdhury, Mehnaz Haq, Hossain Stefania, Asif Khan, Sadman Shoumik Khan

Ahsanullah University of Science and Technology

Bangladesh are unavoidable as transistor size is the most important aspect to be considered by design engineers in the scaling process.

Hence, to solve the scaling concerns, two different paths can be chartered. The first path envisages introduction of new technologies into the conventional planar MOSFET to allow further scaling and boost the performance of scaled CMOS. The second path presupposes adoption of new transistor architectures such as ultra-thin body FETs and multi-gate FETs [3] which inherently have superior electrostatic control over the inversion channel and works at a lower bias voltage.

In this paper, FinFET devices [4], a derivative of multigate FETs have been proposed as a promising alternative over the conventional bulk CMOS-based devices at the nano-scale by providing an overview of comparative analysis of these two structures at a 7-nm nano-scale regime. We know, FinFET based designs impose improved control over short channel effects, low leakage and superior yield below $45 \mathrm{~nm}$ that help to overcome the obstacles in scaling. In this paper, we will demonstrate that FinFET devices offer superior scalability with reduction in gate area, low gate leakage current resulting in higher drain strength and reduced energy consumption by operating at minimum threshold voltage. Furthermore, a detailed simulation study evaluating the performance of the proposed design is presented exhibiting a $2 \mathrm{X}$ increase in drive strength with the application of multiple fins. Fin thickness of $2.725 \mathrm{~nm}$ along with a height of $10.9 \mathrm{~nm}$ has been used in the proposed FinFET structure resulting in an $8 \mathrm{X}$ reduction in gate area which is the smallest 7-nm Fin-FET structure yet developed.

\section{Description and Simulation Approach}

The device schematics of the triple-gate FinFETs modeled in this work are shown in Figure (1). We have used 7-nm FinFET technology by implementing the 7-nm FinFET device model (10nm spacing between edges of the diffusion regions) excerpted from Chen et al. of which the device model extraction and validation are presented in [5]. Synopsys TCAD simulation is used by employing semi-classical transport models with some quantum corrections [6] for analytical process. This device model is constructed by using look-up-tables (LUTs) where the drive strength is calculated utilizing the current equation obtained from [7] and is compatible with SPICE through a Verilog-A interface [5]. 


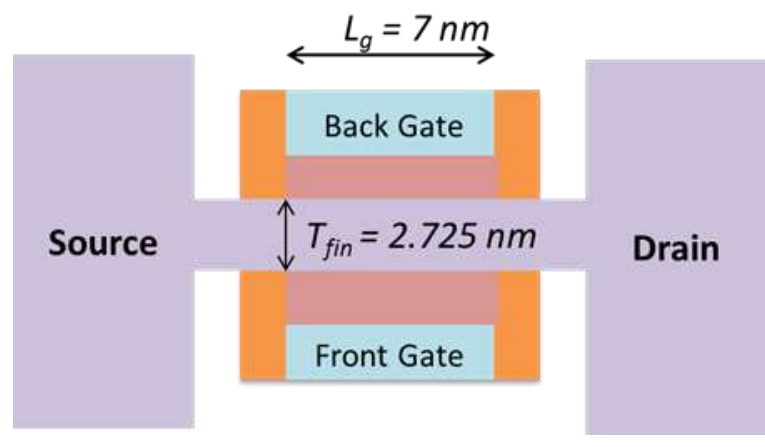

Figure 1. 7-nm FinFET model (top view)

FinFETs are basically 3D structures that rise above the substrate effectively providing more volume than a planar transistor for the same area as a conducting channel. The gate wraps around this thin silicon body called fin, providing better control of the channel and allowing minimum current to leak through the body when the device is in the 'off' state by controlling through the 3 (multiple) gates. This, in turn, enables the use of lower threshold voltages and results in enhanced device performance and less power consumption [8]. In FinFET model, the effective width of the transistor is estimated as $\mathrm{W}_{\text {eff }}=2 \mathrm{H}_{\text {fin }}+\mathrm{T}_{\text {fin }}$ [9]. For this device, the thickness of the fin $\left(\mathrm{T}_{f i n}\right)$ has to be smaller than the gate length $\mathrm{L}_{g}$ so that the short channel effect equations.

TABLE I. EXTRACTED PARAMETERS FOR A 7-NM FINFET MODEL

\begin{tabular}{|c|c|}
\hline Parameter & Value in 7-nm FinFET \\
\hline Physical gate length & $4.905 \mathrm{~nm}$ \\
\hline Oxide thickness & $1.09 \mathrm{~nm}$ (front and back) \\
\hline Body thickness & $2.725 \mathrm{~nm}$ \\
\hline Fin height & $10.9 \mathrm{~nm}$ \\
\hline Fin thickness & $2.725 \mathrm{~nm}$ \\
\hline Fin pitch & $9.725 \mathrm{~nm}$ \\
\hline Source/drain doping & $1 \mathrm{e} 20 / \mathrm{cm} 3$ \\
\hline Source-side underlap & $1.09 \mathrm{~nm}$ \\
\hline Drain-side underlap & $1.09 \mathrm{~nm}$ \\
\hline
\end{tabular}

\section{Results and Discussion}

Based on the methodology presented in Section II and using the 7-nm FinFET model from [5] having a fin thickness of $2.725 \mathrm{~nm}$, we have estimated an overall performance gain of FinFET in nearly every aspect compared to the 7-nm planer CMOS. From our acquired simulations, we can verify that a $34.92 \%$ manifold increased drain current is obtained for 7-nm FinFET compared to the existing 22-nm technology of planer MOSFETs having a minimum gate width of $0.3 \mu \mathrm{m}$. An equivalent 7-nm planer CMOS shows a $28.79 \%$ decreased current with again the minimum gate width of $0.1 \mu \mathrm{m}$ compared to the 7-nm FinFET. Hence the achieved results represent that even if we scale down the conventional MOSFETs to the 7-nm regime we will not be able to reach the equivalent drive strength obtained with just a single fin structure.

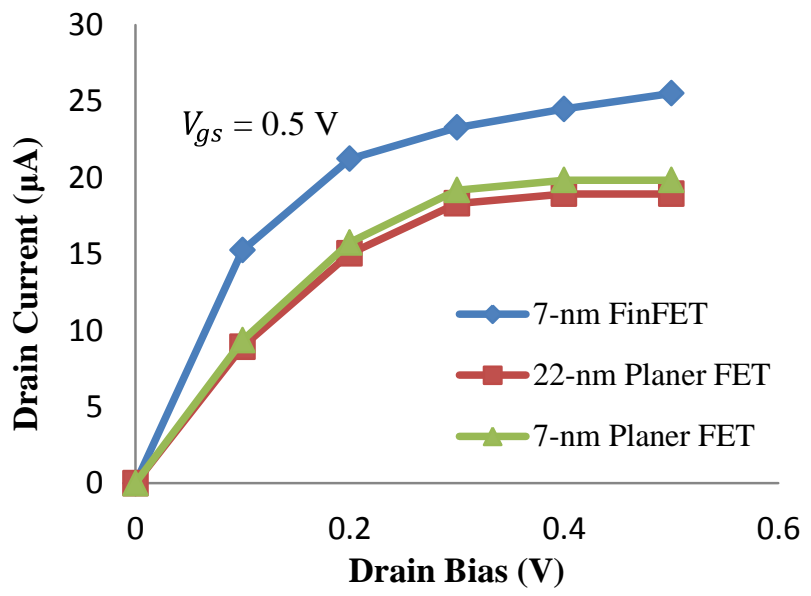

Figure 2. Comparison of drain strength among 7-nm planer, 22-nm planer and 7-nm Fin-FET

As yet, the analysis and all the computations regarding FinFET have been with a single fin structure. Now, rather than a single fin, theoretically by using multiple fins we can obtain an increase in drain current and the corresponding plot clearly shows that there is an almost $50 \%$ increase in the drain current with the increase in the number of fins.

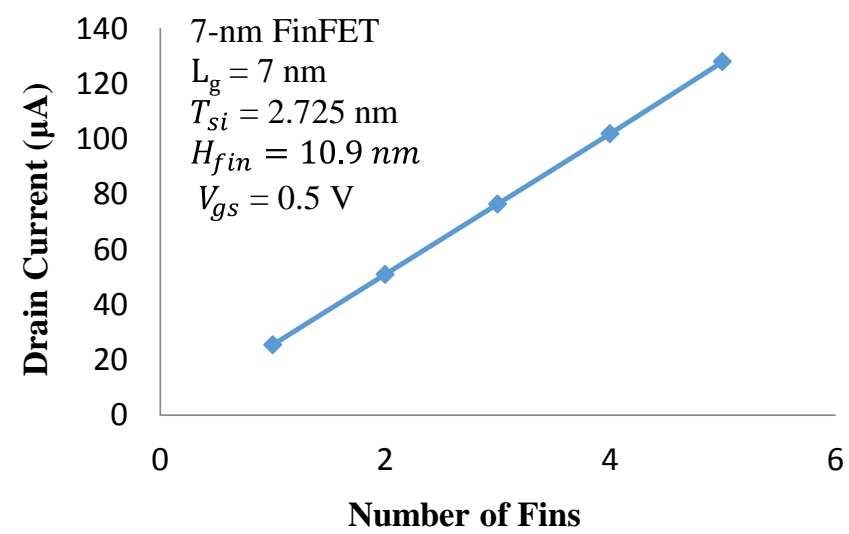

Figure 3. Variation of drain current with increased parallel fins

This clearly surpasses all the past estimations under the 7 $\mathrm{nm}$ nanoscale regime for both planer and tri-gate transistors. Utilizing this availability of increasing drain current with multiple parallel fins we made a comparative analysis of the gate area required in both 7-nm planer and Fin-FETs. While the minimum gate width for a 7-nm planer FET is $100 \mathrm{~nm}$, an equivalent 7-nm FinFET having fin width of $2.725 \mathrm{~nm}$ and a fin pitch of $9.725 \mathrm{~nm}$ can accommodate 8 Fins resulting $204 \mu \mathrm{A}$ drain current which is an increase of $703.1 \%$ more drain strength. This evidently favors the concept of scaling in 
terms of using 7-nm FinFET with the 8X reduction in the gate area.

Earlier, there have been some proposed designs of obtaining increased drain current under the 7-nm regime. But the drive strength obtained from this model exceeds all the previous designs under this nano-scale region.

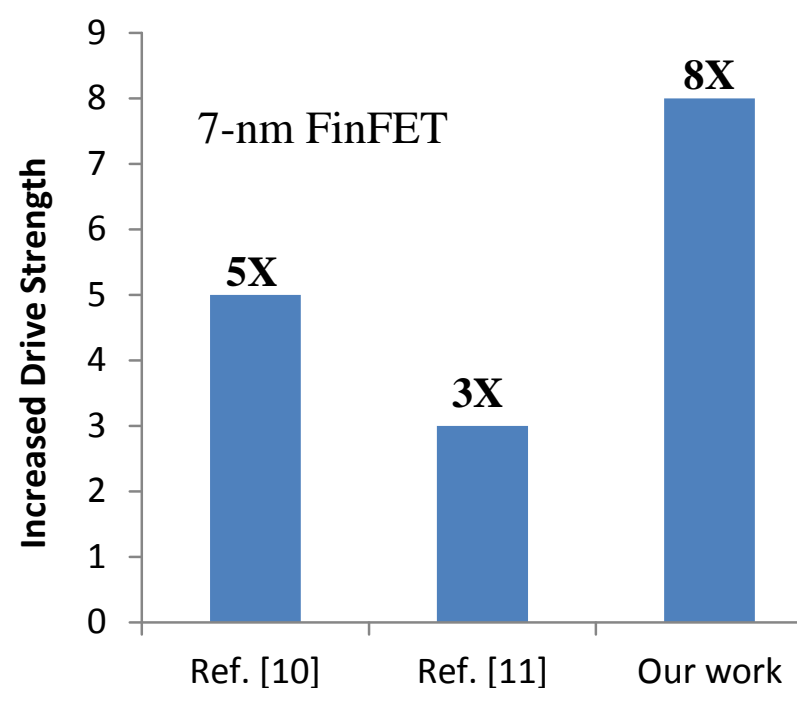

Figure 4. Comparison of drive current among 7-nm FinFET models

Now, as the statistical data shows, FinFET has considerable advantages over planer CMOS and yet the manufacturing challenges make it difficult to go into the fabrication process [12]. As mentioned earlier, this paper contains a relative analysis of both the transistors under the 7-nm regime and henceforth the option of 7-nm planer MOSFET has also been into our consideration. Now, from our simulations we can conclude that we can only obtain the single fin FinFET drain current in figure (2) if the gate width can be minimized to $0.128 \mu \mathrm{m}$ which is $11 \mathrm{X}$ more gate width than the FinFET model. Hence, this precisely does not support the requirements for scaling of MOSFETs.

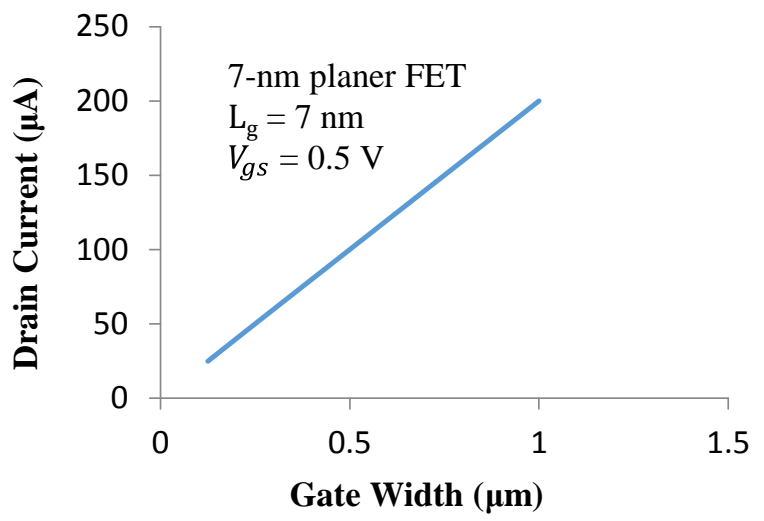

Figure 5. Effect of variation of gate width on drive strength in 7-nm planer transistor.
Apart from decreasing the gate width, an alternative approach to reach the high current obtained in FinFETs can be by varying the supply voltage. For this particular model of FinFET, device simulation data was generated by sweeping gate and drain voltages from $0.1 \mathrm{~V}$ to $0.5 \mathrm{~V}$. Due to linear extrapolation of currents and clamping of capacitances beyond the voltage range $(-0.1$ to $0.8 \mathrm{~V})$ in the Verilog-A model, oscillations may be observed in the simulation output when circuits are simulated close to $0.8 \mathrm{~V}$. That is why, the drain voltage is restricted within the range $0<$ Drain voltage $<0.5 \mathrm{~V}$ [5] in FinFET simulations and this favors the 7-nm planer CMOS as there is no restriction to the maximum sweeping voltage.

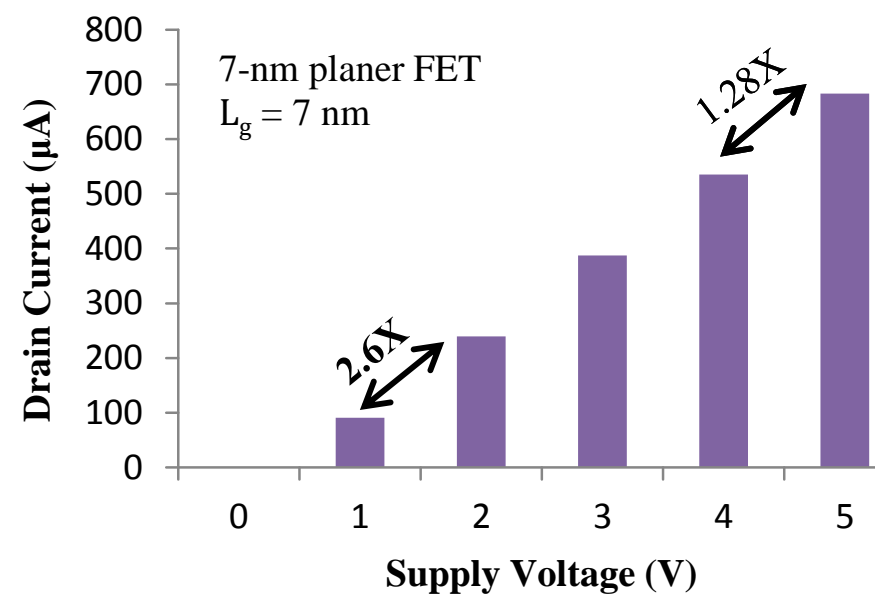

Figure 6. Rate of change of supply voltage in 7-nm planer transistor

And yet, as figure (6) demonstrates with the increase in supply voltage, the rate of drive strength increment reduces counteracting the prospect of increasing the supply voltage for higher drive strength. Moreover, supply voltage scaling is quintessential in order to reduce power density in Integrated Circuits, since increased power density leads to heat which reduces device performance (due to reduced mobility) and further increases standby power consumption (due to increased thermal leakage). Hence, we can state that, neither reduction in gate width nor increasing the supply voltage in case of planer CMOS can reach the drive strength obtained from equivalent 7-nm FinFET.

\section{Conclusion}

This paper shows that after careful optimization FinFETs offer high drain current of $28.79 \%$ over planer CMOS in $7-\mathrm{nm}$ regime that is advantageous in multiple threshold voltage circuits which requires low-power high-performance transistors. FinFET devices can operate at a lower supply voltage than nominal since they have a decreased threshold voltage which significantly improves dynamic power consumption by $10 \%$ as obtained from the supply voltage calculations for a single fin and this constitutes a strong expedition for FinFET adoption. Moreover, the foundation library of FinFET model has the minimum fin height and 
thickness standard values compared to the contemporary 7-nm FinFET models resulting in a gate area reduction of $8 \mathrm{X}$ whereas earlier works proposed a $3 \mathrm{X}$ and $5 \mathrm{X}$ reduction assuring lower dynamic power consumption further. Multiple parallel fins have also been examined and a $2 \mathrm{X}$ increase in the gate current has been noted. Hence, FinFET devices outperform bulk CMOS devices in ultra-low power designs by allowing voltage scalability, lower power consumption and gate area reduction. Overall, since continuous shrinking of channel length increases the speed of devices in very large scale circuits, the FinFET model of this paper is beneficial over planer CMOS for steady miniaturization at a channel length of 7-nm regime.

\section{References}

[1] R. Dreslinski, M. Wiekowski, D. Blaauw, D. Sylvester, and T. Mudge, "Near-threshold computing: reclaiming Moore's law through energy efficient integrated circuits," Proc. of IEEE, Vol. 98, pp 253-256, 2010.

[2] Y. Taur, D. A. Buchanan, W. Chen, D. J. Frank, K. E. Ismail, S. H. Lo, G. A. Sai-Halasz, R. G. Viswanathan, H. J. C. Wann, S. J. Wind, and H.S. Wong, "CMOS Scaling into the Nanometer Regime," Proc. IEEE, vol. 85, pp. 486-504, 1997.

[3] Choi, Yang-Kyu, Daewon Ha, Tsu-Jae King, and Jeffrey Bokor, "Investigation of gate-induced drain leakage (GIDL) current in thin body devices: single-gate ultra-thin body, symmetrical double-gate, and asymmetrical double-gate MOSFETs." Japanese journal of applied physics 42, no. 4S (2003): 2073.

[4] D. Hisamoto, L. Wen-Chin, J. Kedzierski, H. Takeuchi, K. Asano, C. Kuo, E. Anderson, K. Tsu-Jae, J. Bokor, and H. Chenming, (2000) "FinFET - a self-aligned double-gate MOSFET scalable to $20 \mathrm{~nm}$," IEEE Trans. Electron Devices, vol. 47, pp. 2320-2325.

[5] Arun Goud Akkala; Sumeet Kumar Gupta; Sri Harsha Choday; Kaushik Roy (2013), "7nm Si FinFET Models with Symmetric and Asymmetric Underlap for Circuit Simulations," https://nanohub.org/resources/19195.

[6] S. Chen, Y. Wang, X. Lin, Q. Xie, and M. Pedram, "Performance prediction for multiple-threshold $7 \mathrm{~nm}$-FinFET-based circuits operating in multiple voltage regimes using a cross-layer simulation framework," in S3S Conference, Oct. 2014.

[7] M. Zakir Hossain et al., "Electrical Characteristics of Trigate Finfet," Global Journal of Researches in Engineering, Electrical and Electronics Engineering, vol. 11, Issue 7, Version 1.0, Dec. 201.

[8] Sarman K Hadia, Rohit R. Patel, Dr. Yogesh P. Kosta, FinFET Architecture Analysis and Fabrication Mechanism, JCSI International Journal of Computer Science Issues, Vol. 8, Issue 5, No 1, September 2011.

[9] Mishra, P., et al., "FinFET Circuit Design," Nanoelectronic Circuit Design, pp. 23-54, 2011.

[10] T. Cui, et al. "7nm FinFET standard cell layout characterization and power density prediction in near- and super-threshold voltage regimes," in IGCC, 2014.

[11] S. Gupta, V. Moroz, L. Smith, Q. Lu and K. C. Saraswat "7-nm FinFET CMOS design enabled by stress engineering using $\mathrm{Si}, \mathrm{Ge}$, and $\mathrm{Sn}$ ", IEEE Trans. Electron Devices, vol. 61, no. 5, pp.1222 -1230 2014.

[12] Van Dal, Mark J., GeorgiosVellianitis, Ray Duffy, GerbenDoornbos, BartekPawlak, BlandineDuriez, Lhi-Shue Lai et al. "Material aspects and challenges for SOI FinFET integration." ECS Transactions 13, no. 1 (2008): 223-234.
Jamal Uddin Ahmed graduated from BUET, Dhaka, Bangladesh in 1984 in the Department of Electrical and Electronic Engineering. He started his professional life as a teacher in DUET, Bangladesh (then BIT Dhaka) at Gazipur. Latter on he completed his Masters in Computer Engineering form USA. He also worked for 15 years in AMD, HP, Intel designing Microprocessors in USA. Now he is again serving as a teacher in Ahsanullah University of Science and Technology, Dhaka, Bangladesh in the Department of Electrical and Electronic Engineering. In the course of time he published several papers in different journals and conference proceedings. His research interest is in micro-electronics, VLSI, and optical parallel computing.

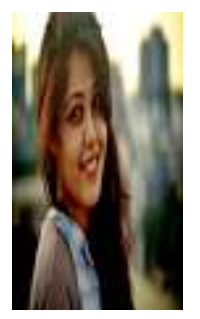

Sarah Nahar Chowdhury has graduated in Electrical and Electronic Engineering of Ahsanullah University of Science and Technology, Bangladesh. She is currently working as the Secretary of Engineering Students' Association of Bangladesh (ESAB). Her research interests include solar cells, renewable energy, organic transistors, nanotechnology and quaternary algebra. She is a student member of Institute of Electrical and Electronics Engineers (IEEE) since 2012.

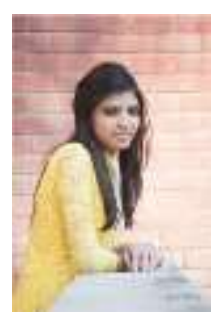

Mehnaz Haq has graduated in electrical and electronic engineering from Ahsanullah University of Science and Technology, Bangladesh. Her research interests include finFET, renewable energy, carbon nanotube transistors, solar cells and nanotechnology. She is a student member of Institute of Electrical and Electronics Engineers (IEEE) since 2013. 
Proc. of The Second Intl. Conf. On Advances In Computing, Control And Networking - ACCN 2015

Copyright (C) Institute of Research Engineers and Doctors, USA .All rights reserved.

ISBN: 978-1-63248-073-6 doi: 10.15224/ 978-1-63248-073-6-14

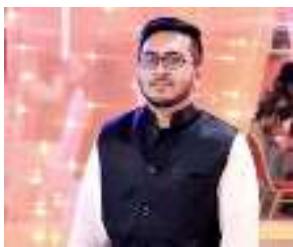

Sadman Shoumik Khan has graduated in Electrical and Electronic Engineering from Ahsanullah University of Science \& Technology, Dhaka, Bangladesh. He believes research is a formalized curiosity to see what everybody else has seen, and to think what nobody else has thought. His research interests include carbon nanotube transistors, renewable energy, nanotechnology and artificial intelligence. He is a student member of Institute of Electrical and Electronics Engineers (IEEE) since 2013.

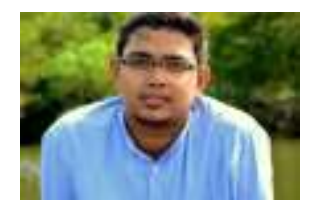

Asif Khan has graduated in Electrical and Electronic Engineering from Ahsanullah University of Science \& Technology, Dhaka, Bangladesh. His research interests include carbon nanotube transistors, renewable energy, transistors. He is a student member of Institute of Electrical and Electronics Engineers (IEEE) since 2013. 